\title{
A Study on the Motif Pattern of Dark-Cloud Cover in the Securities
}

\author{
Jing Long ${ }^{1}$, Wen-Gang $\mathrm{Che}^{1}$, Ren $\mathrm{Yu}^{1}$, Zhi-Yuan Zhou ${ }^{1}$ \\ ${ }^{I}$ Faculty of Information Engineering and Automation \\ Kunming University of Science and Technology Kunming, P.R. China
}

\begin{abstract}
Morphological analysis is the analysis and mining of the graphics formed of the securities price changes. Investors need to forecast the trend of future before buying and selling points, which can avoid great loss. Therefore, the analysis of motif pattern of K-line in the form of futures investment technology analysis is very significant. Based on the thoughts of short-term trend clustering, this paper proposes a method of detecting the motif pattern of Dark-Cloud Cover in stock time series by analysing stock historic data and K-line shape, in order to predict the stock market trends. And we prove the effectiveness and practicality of the method by a series of experimental analysis.
\end{abstract}

\section{Introduction}

The fluctuation of the stock market is a typical time series. In other words, the stock price will change with time. At the point of view of mathematical statistics, it is a series of discrete random data. This orderly random data ( such as $\mathrm{X}_{t}, \mathrm{t}=1,2,3, \ldots, \mathrm{n}$ ) in chronological order is called the time series ${ }^{[1]}$. The stock time series is a sequence related to the data points, including continuous measurement of the stock price on the trading day, that is, the opening price, the closing price, the highest price, the lowest price, the volume and the rate of return. As we all know, the stock market is a huge system that is affected by many factors. It has complex law. And market prices are also changing rapidly. As a comprehensive external form of expression, the financial time series data of stock market contains many objective laws and information. So it is significant to dig useful information from these data, which could help us to better know, master and utilize the law to make forecast, decision and risk management of stocks investment.

At present, morphological analysis is the main method of reversal forecasting of stock price in financial market. ${ }^{[2]}$ It analyses and mines the graphics formed by changes in the securities price, and regard the morphology as best. When the tendency chart of the security price repeated emerge in some morphology, the price will change according to the prior trend, which could predict the trend of future price. Zhou Ding-bin $(2013)^{[3]}$ has published a study of the stock trading operation in the K-line analysis, which used Guizhou 21 stocks as an example, And it illustrated that the methods of morphological analysis impacted the practicality and accuracy of k-line morphology and the stocks trading.
Zhang Dan $(2012)^{[1]}$ has published Reversal Pattern Discovery in Financial Time Series Based on Fuzzy Candlestick Lines, which used a variety of k-line morphology as an example for morphological analysis. It proves the validity of morphological analysis of reversal pattern of mining fuzzy k-line. This paper is based on the ideas that these scholars propose morphological analysis of the stock time series, by combining with short-term tendency theory, we study the motif pattern of Dark-Cloud Cover morphology in the stock time series.

In this paper, the experiment is divided into two parts: the first part is motif pattern that dig Dark-Cloud Cover morphology from the data; the second part is to verify the rewards of investment of Dark-Cloud Cover morphology in the financial market. The experimental data are derived from Qianlong database. Numerical calculation and motif pattern mining of Dark-Cloud Cover morphology are completed by MATLAB software. The main purpose of the study is to dig out the hidden motif pattern from the financial time series, find out the inflection point of the stock price tendency, and evaluate the effectiveness of the motif pattern for the actual investment in the financial market. This could provide reference for investors when they choose stocks buying and selling point. And by assessing the effect of investment income of reversal morphology in the financial market, we can estimate whether or not the research of this kind of reversal morphology is valuable in the stocks time series.

\section{The related definitions}

The models, commonly used by traditional financial time series analysis are mostly a comprehensive description

\footnotetext{
${ }^{*}$ Corresponding author. Email address: wgche@yahoo.com (W.-G. Che)
} 
of the data. For example, an autoregressive model is used to examine the time series. If the time series satisfied the autoregressive model, every time point of the time series applies to the autoregressive relational expression. However, the motif pattern is a local concept. And it reflects more information about a certain aspect of the data, which is about some interesting information fragments on the part of the data. Reversal, as a common language of stock market, refers to that the stock price moves to the opposite direction of the original trend. Specifically, it means that the stock price goes from the bull market to short market or from the short market to bull market. The purpose of this paper is finding out the reversal morphology that the financial market analysts and other investors are interested through the method of data mining, which is also defined as singularity in the financial time series.

Definition 1: Stock Time Series: Given a stock time seriesX, denoted byX $\left(t_{i}, o_{i}, c_{i}, h_{i}, l_{i}\right)$, which $o_{i}, c_{i}, h_{i}, l_{i}$ are used to represent the opening price, the closing price, the highest price and the lowest price of a stock time series at the trade day of $t_{i}$.

Definition 2: Motif pattern: If there are two or more similar segments of $S_{k}=X_{k}\left[t_{i-1}, t_{i+3}\right]$ for $\mathrm{k}=1,2,3, \ldots \mathrm{n}$ within stock time series of $X_{k}$. This similar segment having the same short-term tendency pattern is defined as time series pattern of short-term tendency; that is motif pattern.

$$
S_{k}=X_{k}\left[t_{i-1}, t_{i+1}\right] k=1,2,3, \ldots, n
$$

Definition 3: Reversal morphology: The Dark-Cloud Cover morphology is a kind of top reversal morphology of the motif pattern, which describes the external force that impacts the reversal of the securities price changed trend when the stock price rises to high-order after a certain period of time. The morphology generally appears after the rising trend, and in some cases it may also appear at the top of the horizontal consolidation range.

Definition 4: Reversal point: Taking the closing price of the historical trading data of the stock as the object of investigation and the closing price of the stock will be linked into a line in the short-term tendency, there will be a trading day $t$ of the Dark-Cloud Cover morphology as the reversal point. The significance of the reversal point $t$ is to give investors an early warning that they can sell their stocks to maintain the proceeds or to effectively stop losses when the stock price is turned downward.

Definition 5: Short-term average rate of return: Based on the idea of short-term tendency, a cycle is selected, such as: 3 days, 5 days, 7 days etc. The average price of the closing price of this cycle is computed, and the formula is:

$$
R_{n}=\frac{\sum_{i=1}^{n} \operatorname{close}(i)}{n}(i=1,2,3, \ldots, n)
$$

\section{Dark-Cloud Cover detection and algorithms}

The method of motif pattern detection in Dark-Cloud Cover morphology is based on the short-term tendency clustering theory of time series, and combines with the feature of the Dark-Cloud Cover morphology. The numerical calculation and pattern mining are completed by MATLAB software. In conjunction with definition 3, the following algorithm is available:

Algorithm1: Short-term growth tendency algorithms

Step 1: Take a stock time series of $\mathrm{X}\left(t_{i}, o_{i}, c_{i}, h_{i}, l_{i}\right)$, from any trade day of $i$, compare the closing price of day $i$ and day $i-1$, if $c_{i-1}>c_{i}$, go to step 2; otherwise increase $i$ to next day and go back to step 1 .

Step 2: Compare the stock prices of day $i+1$ and day $i$, if $c_{i+1}>c_{i}$, go to step 3 ; otherwise increase $i$ to next day and go back to step 1 .

Step 3: Compare the stock prices of day $i+2$ and day $i+1$, if $c_{i+2}>c_{i+1}$, go to step 4; otherwise increase $i$ to next day and go back to step 1 .

Step 4: Compare the stock prices of day $i+3$ and day $i+2$, if $c_{i+3}>c_{i+2}$, go to step 5; otherwise increase $i$ to next day and go back to step 1 .

Step 5: A short-term growth tendency is detected. To determine whether there is a Dark-Cloud Cover morphology after growth trend; combining algorithm 2 .

Algorithm2: Dark-Cloud Cover morphology detection algorithm

Take a stock time series of $\mathrm{X}\left(t_{i}, o_{i}, c_{i}, h_{i}, l_{i}\right)$, from any trade day of $i$.

Step 1: respectively compare the opening price $o_{i}$ of day $i$ with the closing price $c_{i-1}$ and the highest price $h_{i-1}$ of dayi -1 , if $o_{i}>c_{i-1}$ oro $o_{i}>h_{i-1}$, go to step 2; otherwise increase $i$ to next day and go back to step 1 .

Step 2: respectively compare the closing price $c_{i}$ of day $i$ with the closing price $c_{i-1}$ and the opening price $o_{i-1}$ of day $i-1$, if $c_{i}>o_{i-1} \& \& c_{i}<c_{i-1}$ or $c_{i}<$ $\frac{c_{i-1}+o_{i-1}}{2}$, go to step 3 ; otherwise increase $i$ to next day and go back to step 1 .

Step 3: If the selected time series transaction day $i$ satisfies stepland satisfies step 2, it is judged that the Dark-Cloud Cover morphology is detected.

Algorithm3: Short-term average rate of return of Dark-Cloud Cover morphology

Calculating the short-term average rate of return is to determine the stock trend that will continue to rise, down or sideways when the Dark-Cloud Cover morphology appears. This gives a warning that the investors should throw or continue to follow up.

First, investors have already held positions for 5 days before Dark-Cloud Cover morphology (a cycle of short-term tendency in this article). We assume that the Dark-Cloud Cover morphology appears on the trading day $i$, then the formula that calculates rate of return of day $i$ and day $i-1$ is recorded as $R^{\prime}$, that is:

$$
R^{\prime}=\frac{\operatorname{close}(i)+\operatorname{close}(i-1)}{2}
$$

And later 5 days after day $i$ as a cycle of short-term tendency, calculate the average return of 5 days trading day after day $i$, denoted by $R^{\prime \prime}$ :

$$
R^{\prime \prime}=\frac{\sum_{i=1}^{5} \operatorname{close}(i)}{5}(i=1,2, \ldots, 5)
$$

Finally, given a standard range $\gamma$, it is determined the stock trend, which is rising, down or sideways after the Dark-Cloud Cover morphology. That is: 


$$
\gamma=\frac{R^{\prime \prime}-R^{\prime}}{R^{\prime}}
$$

Algorithm 4: Motif pattern

Generally, k-line morphology of time series is a pattern that frequently appears in a time sequence, which is very useful to forecast the stock short-term tendencies and prices. In this section, we propose an algorithm that is used to find those motifs of short-term tendency in stock time series.

Step 1: Take a stock time series of $\left(t_{i}, y_{i}\right)$, detect the short-term tendency and remember the time duration.

Step 2: Take another stock time series of $\mathrm{X}\left(t_{i}, y_{i}\right)$, detect the short-term tendency and compare it with former. Then determine whether there are similar segment of time series during same time period. If yes, got to step 3, otherwise go to step 2 .

Step 3: Remember the number of similar segments that is detected and then got back to step 2 .

Step 4: The motifs of the stock time series are detected.

Taking the Shanghai A-stocks as an example, we randomly selected $\mathrm{n}$ stocks, and found the motif pattern of Dark-Cloud Cover morphology in the stock time series by the above algorithm, as shown in "Fig.1, Fig.2, and Fig.3".

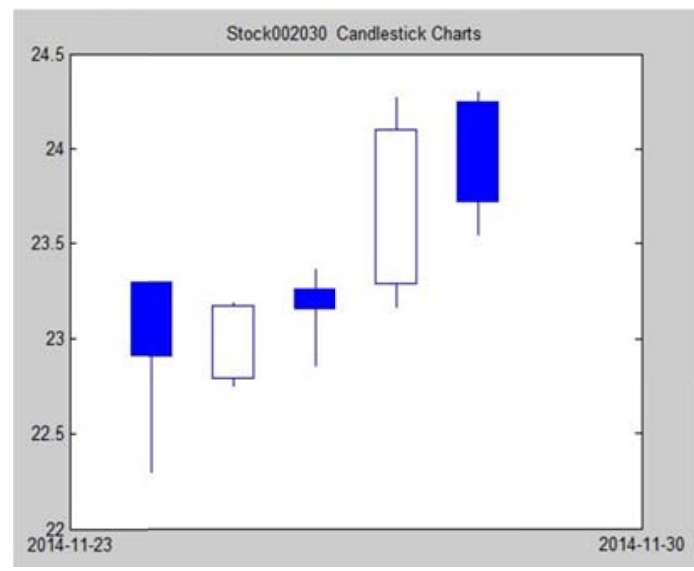

Figure1. Periodicity Dark-Cloud Cover morphology

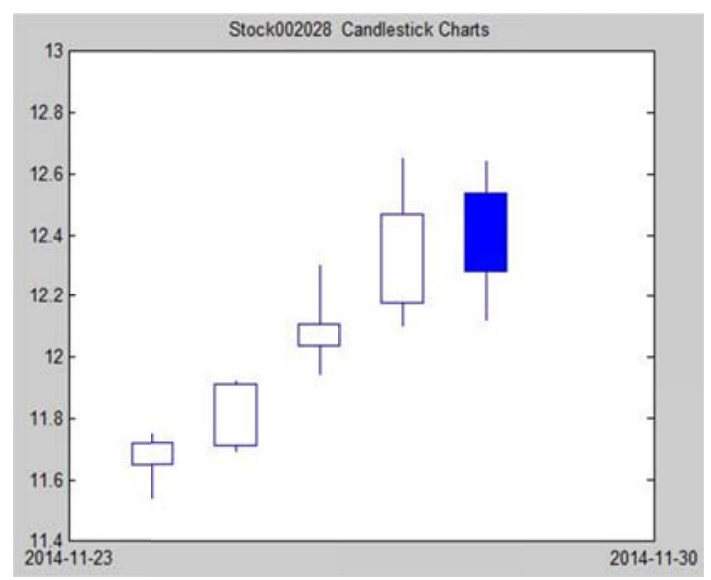

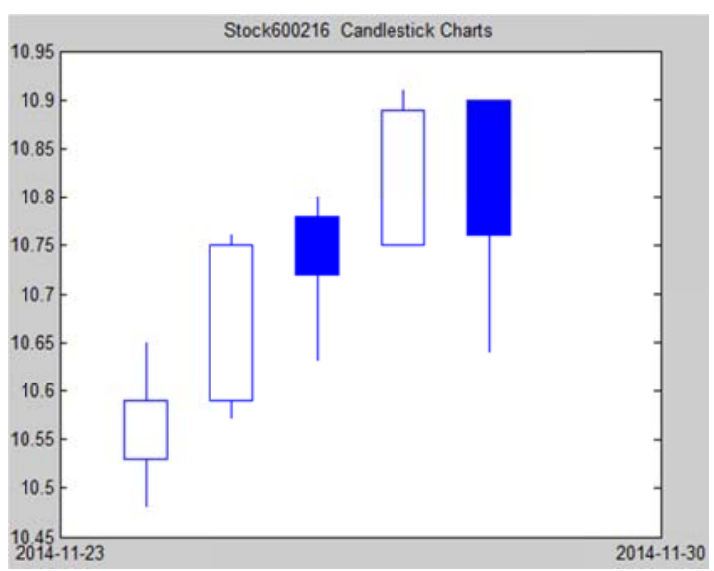

Figure3. Unreversed Dark-Cloud Cover morphology

\section{Empirical Analyses}

Combined with other scholars' research on the factors that influence the fluctuation of securities prices, it is found that there is a tendency in the process of securities price changes. The direction of securities price changes is determined by the current trend. Unless there is an external force that stops or reverses the current trend, the trend will continue. This paper, based on this idea, proposes a meaningful detection method based on motif pattern to detect motif pattern of Dark-Cloud Cover morphology in stock time series. Combining with the short-term tendency theory, and citing the short-term average rate of return, we analysis the short-term trend of Dark-Cloud Cover morphollogy in stock time series, in order to predict the next trend of the stock market, and to give investors an early warning.

In this paper, we selected 200 stocks of Shanghai A-stocks from January 2014 to December 2016 for a period of three consecutive years as the experimental data, which were detected 338 segments of the Dark-cloud cover morphology. This experiment is classified with two quarters as standard of the time period (three months is a quarter), which is divided into a total of six time periods. The data table is shown in Table 1; the time series is shown in "Fig.4"; the moving average is shown in "Fig.5", and the dark is turning point in the Fig.5. In addition, day $i$ and day $i-1$ constitute Dark-cloud Cover morphology.

Table1. The distribution of the number of Dark-Cloud Cover morphology

\begin{tabular}{|c|l|l|l|l|l|l|}
\hline time period & 1 & 2 & 3 & 4 & 5 & 6 \\
\hline $\begin{array}{c}\text { Dark-Cloud } \\
\text { Cover morphology }\end{array}$ & 42 & 80 & 72 & 49 & 43 & 52 \\
\hline
\end{tabular}

Figure2. High Dark-Cloud Cover morphology 


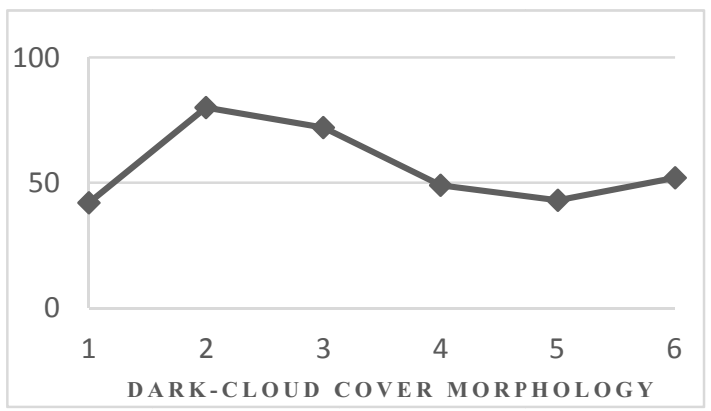

Figure4. Time series diagram

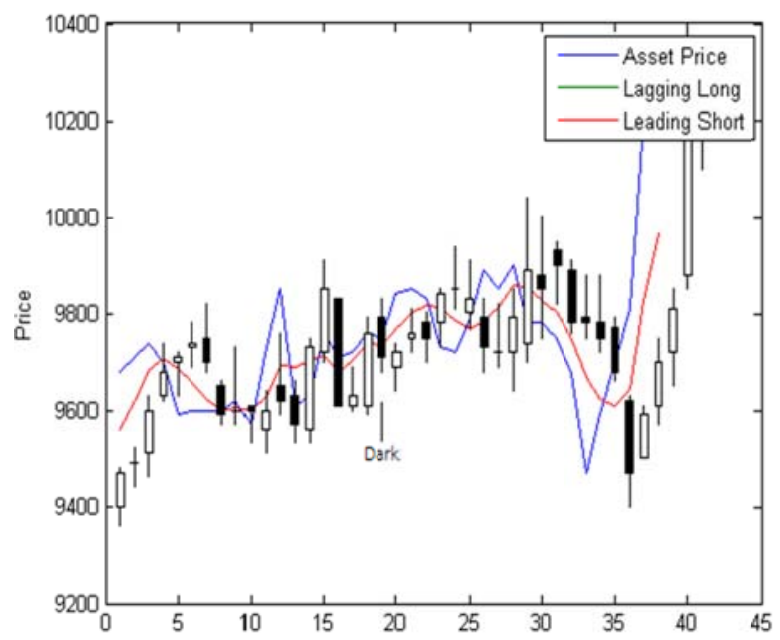

Figure4. The moving average diagram

We get the number and frequency of Dark-Cloud Cover morphology in the Shanghai A shares in January 2014 to December 2016 for three consecutive years by analyzing the data table. However, if we want to better to determine the follow-up trend of the stock by using Dark-Cloud Cover morphology, we need to combine short-term Trends, with calculating the short-term average returns and ranges. This experiment collects the closing price of trading day of Dark-Cloud Cover morphology and the closing price of the next five days after Dark-Cloud Cover morphology. The results calculated by the formula (4) are combined with the collected data. In this paper, the standard range $\gamma$ of the stock trend in the late stage of Dark-Cloud Cover morphology is setting up as $2 \%$. The data statistics are shown in Tables 2 and 3 (Table 2 shows the distribution of the late trend of the stock; Table 3 shows the ratio of the stock overall trend)

Table2. The distribution of the late trend of the stock

\begin{tabular}{|c|l|l|l|l|l|l|}
\hline Time period & 1 & 2 & 3 & 4 & 5 & 6 \\
\hline rise & 2 & 7 & 6 & 2 & 2 & 2 \\
\hline down & 35 & 48 & 44 & 35 & 34 & 47 \\
\hline sideways & 5 & 25 & 22 & 12 & 7 & 3 \\
\hline
\end{tabular}

Table3. The ratio of the stock overall trend

\begin{tabular}{|c|c|c|c|}
\hline Stock trend & rise & down & sideways \\
\hline \multirow{2}{*}{ gross ratio } & 21 & 243 & 74 \\
\cline { 2 - 4 } & $6.21 \%$ & $71.90 \%$ & $21.89 \%$ \\
\hline
\end{tabular}

By analyzing the data in Table 3 , it can be concluded that the number of stocks down accounted for more than $71.90 \%$, sideways trend accounted for $21.89 \%$, and the rise trend that was the lowest, accounted for only $6.21 \%$ of the gross ratio. So the possibility of the stock down is largest when Dark-Cloud Cover morphology appears, which provides reference for investors to sell the timing, and reminds investors should not blindly chase high to avoid inflection point of stock price in a certain period of time. Besides, it is more effective to protect the vested income of investors.

\section{Conclusions}

Based on the idea of short-term trend, this paper proposes a method to detect motif pattern of singular morphological fragments in stock time series. Through the detection and analysis of Shanghai A stocks data, the results show that we can achieve better results by using the method to analysis the morphological of stock time series and the late trend. Finally, though the analysis of the closing price corresponding to the motif pattern of singularity, the validity of the method proposed in this paper is further confirmed. Of course, there are many factors that affect the stock price, this article only analyses the closing price. In the later period, we will analyze the volume of the stock market, which hope to achieve the expected results.

\section{References}

1. Qiu-jun Lan, Dan Zhang, Long-ling Xiong. Reversal Pattern Discovery in Financial Time Series Based on Fuzzy Candlestick Lines. In: International Symposium on Complex Systems Theory \& Applications. Changsha, 2011

2. De-hong Liu. Technical analysis of stock investment. Beijing: Economic management press.2009,pp-78-146(in Chinese)

3. Ding-bin Zhou, Jie Wang. study of the stock trading operation in the K-line analysis. Economics and management science 2013, (10) (in Chinese).

4. Engle RF. Autoregressive conditional heteroscedasticity with estimates of the variance of United Kingdom inflation. Econometrical 
Journal of the Econometric Society,

1982(6),pp-987-1007

5. Lee C-H L, Liu A. Pattern discovery of Fuzzy Time Series for financial prediction. IEEE

Transactions on Knowledge and Data Engineering, 2006, 18(5),pp-613-624

6. Xiao-di Liu, Wen-gang Che, and Qing-jiang Zhao, "Analysis of outliers and public information arrivals using wavelet transform modulus Maximum," The 2nd IEEE International Conference on Information and Financial Engineering, September 2010, pp-176-179.

7. Qiang $\mathrm{Fu}$, Wen-gang Che, "Study on isolated singularity data mining algorithm based on sliding window," JINGXI SCIENCE, vol. 29, pp-273-276, April 2011(in Chinese).

8. Yue Zhang, Jie Liu, and Hang Li. An outlier detection algorithm based on clustering analysis, The 1st International Conference on Pervasive Computing, Signal Processing and Applications, 2012,pp-1126-1128. 